\title{
HIV Vulnerability in Out-of-School Adolescents and Youth in Yunnan, China
}

\author{
Jianghong Li ${ }^{1,2}$, Lijun Yang ${ }^{3}$, Zhouyang Ren ${ }^{3}$, Chongbin $\mathrm{Mo}^{3}$, Dingwen Chen ${ }^{3}$, Fuqiang Dai ${ }^{3}$, \\ Mingzhong Jiang ${ }^{3}$, Zhijie Tang ${ }^{3}$, Peter Jacoby ${ }^{2}$ \\ ${ }^{1}$ Curtin Health Innovation Research Institute, Centre for Population Health Research, Curtin University, Perth, Australia; ${ }^{2}$ Telethon \\ Institute for Child Health Research, The Centre for Child Health Research, The University of Western Australia, Perth, Australia; \\ ${ }^{3}$ Criminal Science and Technology Key Laboratory, Yunnan Provincial Police Officers Academy, Kunming, China. \\ Email: jianghongl@ichr.uwa.edu.au
}

Received March 14 ${ }^{\text {th }}, 2012$; revised April 17 ${ }^{\text {th }}, 2012$; accepted April 26 $6^{\text {th }}, 2012$

\begin{abstract}
This study investigated multiple aspects of vulnerability to HIV in out-of-school adolescents and youth in Yunnan Province, a high HIV risk region in China. The findings show that socially disadvantaged adolescents and youth were overrepresented in the out-of-school young people in Yunnan. The out-of-school young people in Yunnan exhibited 1) limited knowledge about HIV transmission and prevention, 2) a high prevalence of unprotected sexual activity, 3) high exposure to illicit drugs and alcohol use and a high prevalence of drug use in themselves, and 4) limited access to health services. There is an indication of higher exposure to risk behaviours in the younger age group. The study population used multiple media, particularly television, internet and radio, to obtain information about HIV transmission and prevention, particularly in the younger age group. These media may be an effective way of reaching this population in future HIV education and prevention programs in the region.
\end{abstract}

Keywords: HIV/AIDS Education and Prevention; Vulnerability; Out-of-School Adolescents and Youth; Yunnan; China

\section{Introduction}

Youth and adolescents are a primary population group at high risk for HIV/AIDS. In 2007, the total number persons infected with HIV reached 33.2 million: of these about 10 million cases were found in those 15 to 24 years of age and 2.5 million cases in those below age 15 [1].

While the overall HIV/AIDS prevalence remains low in China, there are regions in Yunnan Province where the prevalence is high and fast growing, with the epidemic spreading from high risk groups to the general population [2]. Yunnan Province is most affected by the HIV/AIDS with the highest number of HIV infections and AIDS patients in China (Figures 1 and 2) [3].

By the end of September 2008, the cumulative cases of HIV/AIDS had reached 63,322 , making up $24 \%$ of the total of HIV/AIDS patients in China [4]. Adolescents and youth are increasingly affected by the spread of the HIV/AIDS epidemic in China. By the end of 2007, $0.05 \%$ of China's total population were infected with HIV, but this increased to $0.07 \%$ of the population between 14 and 49 years of age [5]. Based on the Yunnan Provincial official statistics for 2007, more than $80 \%$ of the total HIV/AIDS cases were found in persons below age 30 [5].

Adolescents and youth have strong curiosity, which is the most common reason for initiating drug use in adolescents in Yunnan [6]. They undergo a major physiological and psychological change before reaching adulthood, and have a high tendency for risk taking behaviours. Hence they are most at risk of contracting HIV. Educating this population group about the transmission and harm of HIV/AIDS is thus the most important way of protecting them from HIV/AIDS. The Chinese Government considers as a high priority HIV education and prevention programs that target adolescents and youth. Such programs have been incorporated into the curriculum plans at all school levels (primary school, secondary school and university).

However, adolescents and youth who have left the formal educational system are not covered by the current HIV educational programs in China. This is an important gap in China's current HIV/AIDS prevention programs. Due to a lower level of economic development and a higher poverty rate, the rate of school nonattendance and drop out among adolescents and youth is much higher in Yunnan than in the nation as a whole [7]. Previous research in China has shown that out-of-school adolescents 


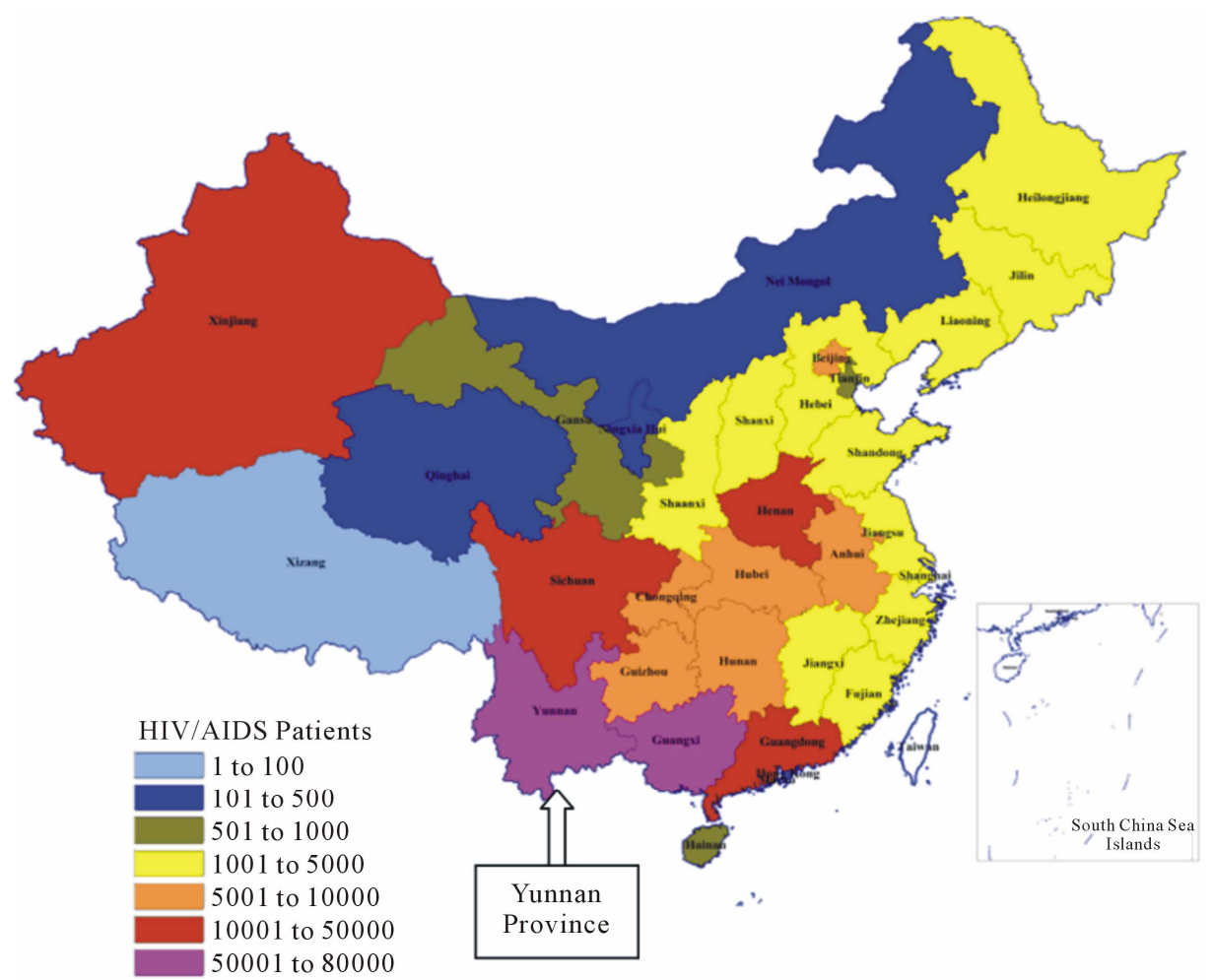

Figure 1. Cumulative reported HIV and AIDS patients in China as of the end of September 2011: cited and modified from “An assessment of China's HIV/AIDS Epidemic in 2011” (p. 10) by China Ministry of Health, UNAIDS, and WHO, November 2011 in Beijing [3].

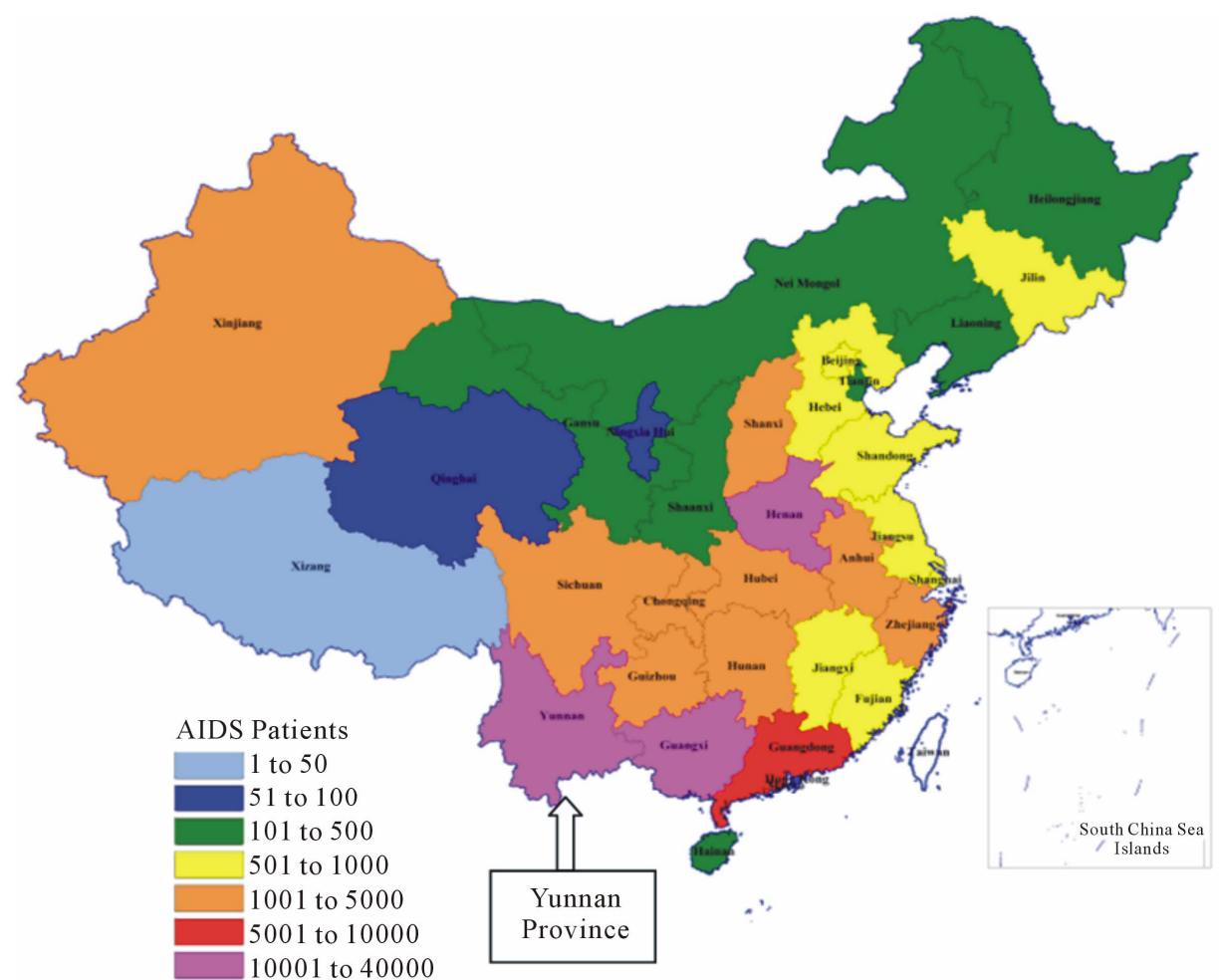

Figure 2. Cumulative reported AIDS patients in China as of the end of September 2011: cited and modified from the same source as in Figure 1. 
and youth have several disadvantages and vulnerabilities [8]. With no or a low level of schooling, there are limited sources where they can obtain accurate and adequate information about health in general and HIV in particular. They live in a social environment where traditional social control mechanism through local communities is much weakened and hence they have little guidance and fewer constraints for risky behaviours that contribute to HIV/ AIDS transmission, such as frequent and unprotected sexual activities, drug and alcohol abuse. Such vulnerability is augmented by limited knowledge about HIV transmission and prevention and limited access to health services [9-11].

Yet to date there is little research in Yunnan that has been conducted on HIV vulnerability in out-of-school adolescents and youth. This study aimed to provide reliable evidence for developing future HIV prevention and education programs targeting out-of-school young people in Yunnan Province. It described the social and demographic profiles of out-of-school adolescents and youth in Yunnan, their vulnerability to HIV, with respect to knowledge about HIV transmission paths, risk taking behaviours (unprotected sexual activity and drug abuse) and access to and use of health information and services.

\section{Methods}

\subsection{Study Population}

Currently, there is no consensus about defining "adolescents and youth" in research on HIV risk and vulnerability. For example, Dick, Ferguson and Ross defined those aged between 10 and 24 as "young people" at HIV risk: ages 12 - 19 as adolescents and ages 15 - 24 as youth, whereas, others define young people as those aged between 15 and 24 and target them as the primary high risk group [12]. We took the following factors into consideration in defining "out-of-school adolescents and youth", namely our study population. The terms "young people" and "adolescents and youth" will be used interchangeably in the paper. We employed the definition of adolescence by Britannica Concise Encyclopedia: adolescence is the "period of life from puberty to adulthood (approximately ages 12 - 20) characterized by marked physiological changes, development of sexual feelings, efforts toward the construction of identity, and a progression from concrete to abstract thought” [13]. Our definition of youth includes those youngsters 21 through to 24 years of age. In defining being "out-of-school", we have taken into account both compulsory (primary school and junior high school) and non-compulsory schooling (senior high to university) in China. Children commence primary schooling at age 7 for 6 years, at age 13 for junior high school for 3 years, at age 16 for senior high school for 3 years, and normally at age 19 - 21 for a university degree for 4 years. Some high shool graduates enter university later than 19 years of age because they do not pass the university entrance examination in the first year and need additional 1 to 2 years to take the examination again. The data collection targeted adolescents and youth who were at school ages (12 to 24) but who were not enrolled in a school. These young people are likely to be vulnerable not only because they are going through rapid physiological and psychological transitions but also because they tend to be only tenuously attached to the labour market due to truncated education and lack of life skills. Hence they live on the margin of the society and float out of social control mechanisms.

The study population included 248 adolescents and youth 12 to 24 years of age, who were not enrolled in school and residing in three municipalities of Yunnan Province: Kunming, Ruili and Dali. Ruili is located in southern Yunnan, borders with Burma, and has the highest HIV prevalence in Yunnan. Kunming (provincial capital, located in central Yunnan) and Dali (located in the western Yunnan) have a moderate prevalence of HIV (Figure 3).

\subsection{Data Collection}

Data collection took place between June and August 2009. A random sampling was used to select participants from two communities (one urban and one rural) in each of the three municipalities. With the assistance of the residential committee of the selected urban communities, the local youth leaders of the sampled rural communities, a list of adolescents and youth who were out-of-school at the time of data collection was compiled. From this list, every two persons on the list were randomly selected for the study, with an intended a sex ratio of three males to one female, which was consistent with the sex ratio of

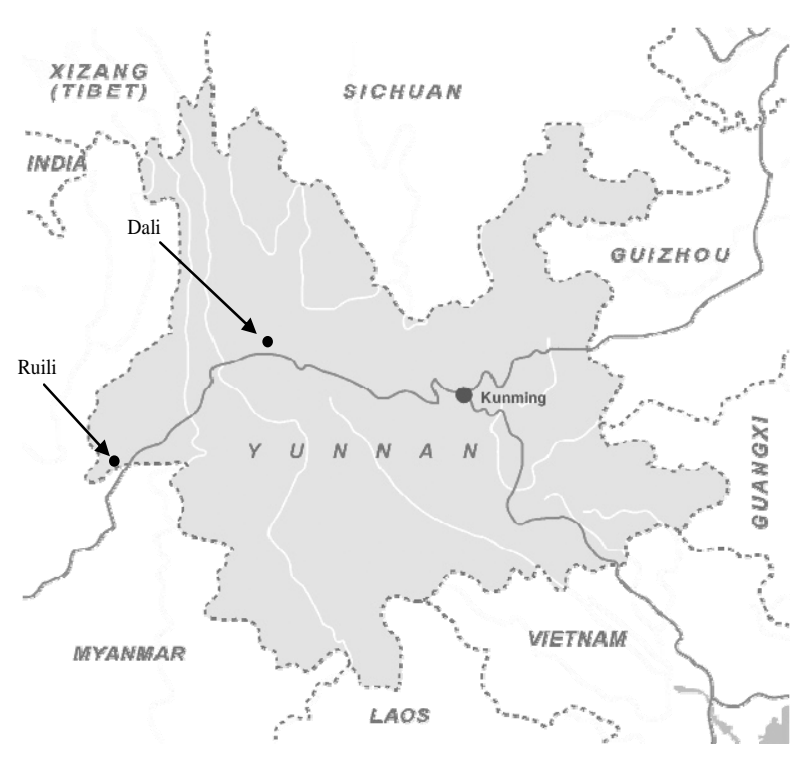

Figure 3. Yunnan's prefectures and regions. 
the HIV/AIDS patients reported in the provincial statistics as of the end of 2008 [4]. In total 289 out-of-school adolescents and youth were selected for the study and to answer the questionnaire administered by trained interviewers. Two hundred and forty nine persons completed the questionnaire: 80 in Kunming, 84 in Ruili and 85 in Dali. In total 40 people declined to answer the questionnaire: out of these more were males than females and as a result, the sex ratio of the effective sample (249) was lower than the intended ratio ( 3 males to 1 female).

The questionnaire included questions about the sociodemographic characteristics of the participants, their family background, drug and alcohol use in parents, participants' levels of knowledge about harms related to illicit drugs and HIV/AIDS, sexual behaviours and condom use, drug use, alcohol consumption, smoking and access to and use of preventative services. The questionnaire was piloted prior to its administration and on average it took 25 minutes to complete.

Prior to completing the questionnaire, the researchers had explained the purpose and the nature of the study to the participants and sought their consent to participate. The participants were informed that they were free to leave the study at any time without providing the researchers with any reasons. The study was approved and funded by the Chinese Government and the UNICEF. Its conduct was monitored and reviewed by the HIV/AIDS Prevention and Control Office of Yunnan Provincial Health Bureau.

We considered the following risk factors for vulnerability to HIV in young people: lack of or poor education, unemployment, poverty, poor or lack of knowledge about HIV/AIDS transmission prevention, limited access to health services and commodities, early sexual debut (before age 15), unprotected sex, multiple partners, and drug and alcohol use [12].

\section{Results}

\subsection{Sociodemographic Characteristics of the Study Population}

The average age of the respondents was 19.7 years of age, with a standard deviation of 2.3 (Table 1). About $70 \%$ of the respondents were male and 30\% were females; more than $50 \%$ of them were national minorities (56\%) and the majority (70.2\%) lived in rural areas. About $9 \%$ of the sample had primary schooling, 50\% had junior high degree, around $31 \%$ completed senior high schooling or a trade certificate, and about $10 \%$ had a diploma or a higher degree. A significant proportion of the sample was either not in the labour market or only tenuously attached to it: $21 \%$ were unemployed and about $18 \%$ were in short-term employment. About 32\% were farm labour,
Table 1. Socio-demographic characteristics of the study population $(\mathrm{N}=\mathbf{2 4 8})$.

\begin{tabular}{|c|c|c|}
\hline Variable name & $\mathrm{N}$ & $\%$ \\
\hline Mean age (STD; range) & \multicolumn{2}{|c|}{$19.72(2.33 ; 12-24)$} \\
\hline \multicolumn{3}{|l|}{ Gender } \\
\hline Male & 173 & 69.8 \\
\hline Female & 75 & 30.2 \\
\hline \multicolumn{3}{|l|}{ Nationality } \\
\hline Han Chinese & 110 & 44.4 \\
\hline Dai & 80 & 32.3 \\
\hline Bai & 42 & 16.9 \\
\hline Other & 17 & 6.4 \\
\hline \multicolumn{3}{|l|}{ Residence } \\
\hline Urban & 50 & 20.1 \\
\hline Suburban & 24 & 9.6 \\
\hline Rural & 174 & 70.2 \\
\hline \multicolumn{3}{|l|}{ Education $^{\mathrm{a}}$} \\
\hline Primary school & 22 & 8.9 \\
\hline Junior high & 124 & 50.0 \\
\hline Senior high/trade school & 76 & 30.6 \\
\hline Diploma and higher & 24 & 9.7 \\
\hline \multicolumn{3}{|l|}{ Employment } \\
\hline Unemployed & 52 & 21.0 \\
\hline Service & 54 & 21.8 \\
\hline Short term employment & 44 & 17.7 \\
\hline Farmer/labour & 79 & 31.9 \\
\hline Other $^{\mathrm{b}}$ & 19 & $7.6 \mathrm{~s}$ \\
\hline \multicolumn{3}{|l|}{ Parents' marital status } \\
\hline Living together & 217 & 87.5 \\
\hline Separated/divorced & 20 & 8.1 \\
\hline Passed away/don't know & 11 & 4.4 \\
\hline \multicolumn{3}{|l|}{ Father's education } \\
\hline Never been to school ${ }^{\mathrm{C}}$ & 50 & 20.1 \\
\hline Primary school & 63 & 25.4 \\
\hline Junior high & 87 & 35.1 \\
\hline Senior high/trade school & 31 & 12.5 \\
\hline Diploma and higher & 17 & 6.9 \\
\hline \multicolumn{3}{|l|}{ Mother's education } \\
\hline Never been to school & 63 & 25.4 \\
\hline Primary school & 83 & 33.5 \\
\hline Junior high & 66 & 26.6 \\
\hline Senior high/trade school & 22 & 8.9 \\
\hline Diploma and higher & 14 & 5.6 \\
\hline \multicolumn{3}{|l|}{ Living arrangement } \\
\hline Live with parents & 173 & 69.8 \\
\hline Rent by self & 50 & 20.2 \\
\hline Other & 25 & 10.0 \\
\hline \multicolumn{3}{|l|}{ Financial support } \\
\hline Parents & 120 & 48.4 \\
\hline Self & 113 & 45.6 \\
\hline Relatives, friends/other & 15 & 6.0 \\
\hline \multicolumn{3}{|l|}{ Reasons for leaving school } \\
\hline Finished school & 113 & 45.6 \\
\hline Don't want to go to school & 61 & 24.6 \\
\hline Scores not high enough for university & 33 & 13.3 \\
\hline Financial reasons & 21 & 8.4 \\
\hline Other reasons & 20 & 8.1 \\
\hline
\end{tabular}

${ }^{\mathrm{a}}$ Missing cases $(\mathrm{n}=2)$; ${ }^{\mathrm{b}}$ Other: small/family business $(\mathrm{n}=10)$, government firms $(n=3)$, professional $(n=2)$ and students not in formal school $(n=4)$; 'Including respondents who did not know their parents' education level (4.4\% for father, $5.2 \%$ for mother). 
which is considered as the lowest occupational status in China.

The vast majority of the respondents reported that their parents $(87.5 \%)$ were married and living together. About $20 \%$ of the respondents had a father with no formal schooling, and in $25.4 \%$ of them the father had only primary schooling. The corresponding figures for maternal schooling are $25.4 \%$ and $33.5 \%$ respectively. Despite that about $70 \%$ of the respondents were living with their parents, only $48.4 \%$ of them received financial support from parents, and $45.6 \%$ supported themselves or received support from relatives, friends or others. A considerable proportion of the respondents were living on their own or with others (30.2\%). While about $46 \%$ of the respondents left the school because they had completed a degree, $25 \%$ of them left the school because they did not want go to school, and $16.5 \%$ did so for financial or other reasons.

\subsection{Exposure to Illicit Drugs and Alcohol}

While $4.4 \%$ of the respondents reported that their parents used illicit drugs, more than three times as many (18\%) reported that one or both parents drank excessively sometimes and, in 9\% of them one parent often drank excessively (Table 2). These percentages are higher in younger respondents (age 12 - 20) than in the older age group (age 21 - 24). There were more than three times as many respondents who had seen other drugs (K powder, ecstasy, opium and ice) than heroin which has been commonly used in Yunnan over the last two decades. In the 12 - 20 age group between 21\% and 33\% and in the 21 - 24 age group between $25.8 \%$ and $35.5 \%$ had seen other drugs than heroin. In 12 - 20 year olds, more people had seen heroin (11\%) and ecstasy (32.9\%) than in the 21 24 year olds (7.7\% for heroin and 30.2\% for ecstasy). About $20 \%$ of the respondents knew friends who used drugs: more so in the older (25.8\%) than in younger (16.8\%) respondents.

\subsection{Risk Taking Behaviours}

About half of the respondents smoked in the year prior to the data collection in 2009 (49\% for 12 - 20 year olds and $48.4 \%$ for age group 21 - 24) (Table 3). Nearly one third of the respondents drank alcohol in the last year: while less than $10 \%$ of them became drunk all the time, more than 30\% were drunk sometimes (31.6\% for age 12 - 20 and 39.8\% for age 21 - 24). About 3\% of the 12 - 20 age group had used drugs and twice as many had done so in the 20 - 24 year olds. However, the younger age group (5.8\%) was almost twice as likely as the older age group (3.2\%) to report they were not sure if they had taken drugs before. About 33\% of the 12 - 20 year olds had sex and among them $15.7 \%$ had their sexual debut before age
Table 2. Exposure to drugs and alcohol.

\begin{tabular}{|c|c|c|c|}
\hline Age $12-20(n=155)$ & $\mathrm{N}$ & $\%$ & $\begin{array}{c}\text { 95\% Confidence } \\
\text { Interval (CI) } \\
\end{array}$ \\
\hline \multicolumn{4}{|l|}{ Parents use drugs } \\
\hline No & 148 & 95.5 & $92.2-98.8$ \\
\hline Yes & 7 & 4.5 & $1.2-7.8$ \\
\hline \multicolumn{4}{|l|}{ Parents drink excessively } \\
\hline Neither drink & 112 & 72.3 & $65.3-79.3$ \\
\hline $\begin{array}{l}\text { One parent often drink } \\
\text { excessively }\end{array}$ & 15 & 9.7 & $5.0-14.4$ \\
\hline $\begin{array}{l}\text { One or both sometimes } \\
\text { drink excessively }\end{array}$ & 28 & 18.1 & $12.0-24.2$ \\
\hline \multicolumn{4}{|l|}{ Have seen drugs } \\
\hline Heroin & 17 & 11.0 & $6.1-15.9$ \\
\hline K powder & 33 & 21.3 & $14.9-27.7$ \\
\hline Ecstasy & 51 & 32.9 & $25.5-40.3$ \\
\hline Opium & 49 & 31.6 & $24.3-38.9$ \\
\hline Ice & 43 & 27.7 & $20.7-34.7$ \\
\hline \multicolumn{4}{|l|}{ Know friends who use drugs } \\
\hline Yes & 26 & 16.8 & $10.9-22.7$ \\
\hline No & 101 & 65.2 & $57.7-72.7$ \\
\hline Not sure & 28 & 18.0 & $12.0-24.0$ \\
\hline Age $21-24(n=93)$ & $\mathrm{N}$ & $\%$ & $95 \% \mathrm{CI}$ \\
\hline \multicolumn{4}{|l|}{ Parents use drugs } \\
\hline No & 89 & 95.7 & $91.6-99.8$ \\
\hline Yes & 4 & 4.3 & $1.2-10.6$ \\
\hline \multicolumn{4}{|l|}{ Parents drink excessively } \\
\hline Neither drink & 70 & 75.3 & $66.5-84.1$ \\
\hline $\begin{array}{l}\text { One parent often drink } \\
\text { excessively }\end{array}$ & 7 & 7.5 & $2.1-12.9$ \\
\hline $\begin{array}{l}\text { One or both sometimes } \\
\text { drink excessively }\end{array}$ & 16 & 17.2 & $9.5-24.9$ \\
\hline \multicolumn{4}{|l|}{ Have seen drugs } \\
\hline Heroin & 2 & 2.2 & $0.3-7.6$ \\
\hline K powder & 27 & 29.0 & $19.8-38.2$ \\
\hline Ecstasy & 24 & 25.8 & $16.9-34.7$ \\
\hline Opium & 33 & 35.5 & $25.8-45.2$ \\
\hline Ice & 28 & 30.1 & $20.8-39.4$ \\
\hline \multicolumn{4}{|l|}{ Know friends who use drugs } \\
\hline Yes & 24 & 25.8 & $16.9-34.7$ \\
\hline No & 48 & 51.6 & $41.4-61.8$ \\
\hline Not sure & 21 & 22.6 & $14.1-31.1$ \\
\hline Total & $\mathrm{N}$ & $\%$ & $95 \% \mathrm{CI}$ \\
\hline \multicolumn{4}{|l|}{ Parents use drugs } \\
\hline No & 237 & 95.6 & $93.0-98.2$ \\
\hline Yes & 11 & 4.4 & $1.8-7.0$ \\
\hline \multicolumn{4}{|l|}{ Parents drink excessively } \\
\hline Neither drink & 182 & 73.4 & $67.8-79.0$ \\
\hline $\begin{array}{l}\text { One parent often drink } \\
\text { excessively }\end{array}$ & 22 & 8.9 & $5.3-12.5$ \\
\hline $\begin{array}{l}\text { One or both sometimes } \\
\text { drink excessively }\end{array}$ & 44 & 17.7 & $12.8-22.6$ \\
\hline \multicolumn{4}{|l|}{ Have seen drugs } \\
\hline Heroin & 19 & 7.7 & $4.3-11.1$ \\
\hline K powder & 60 & 24.2 & $18.7-29.7$ \\
\hline Ecstasy & 75 & 30.2 & $24.4-36.0$ \\
\hline Opium & 82 & 33.1 & $27.1-39.1$ \\
\hline Ice & 71 & 28.6 & $22.8-34.4$ \\
\hline \multicolumn{4}{|l|}{ Know friends who use drugs } \\
\hline Yes & 50 & 20.2 & $15.1-25.3$ \\
\hline No & 149 & 60.1 & $53.9-66.3$ \\
\hline Not sure & 49 & 19.7 & $14.6-24.8$ \\
\hline
\end{tabular}


Table 3. Risk-taking behaviours.

\begin{tabular}{|c|c|c|c|}
\hline Age 12 - $20(n=155)$ & $\mathrm{N}$ & $\%$ & $95 \% \mathrm{CI}$ \\
\hline \multicolumn{4}{|l|}{ Ever smoked before: } \\
\hline Yes & 75 & 48.4 & $40.5-56.3$ \\
\hline No/unknown & 80 & 51.6 & $43.7-59.5$ \\
\hline \multicolumn{4}{|l|}{ Smoked last year } \\
\hline Yes & 76 & 49 & $41.1-56.9$ \\
\hline No & 79 & 51 & $43.1-58.9$ \\
\hline \multicolumn{4}{|l|}{ Ever drank before } \\
\hline Yes & 93 & 60 & $52.3-67.7$ \\
\hline No & 62 & 40 & $32.3-47.7$ \\
\hline \multicolumn{4}{|l|}{ Drank last year } \\
\hline Yes & 43 & 27.7 & $20.7-34.7$ \\
\hline No & 112 & 72.3 & $65.3-79.3$ \\
\hline \multicolumn{4}{|l|}{ Ever got drunk } \\
\hline Yes all the time & 9 & 5.8 & $2.1-9.5$ \\
\hline Yes sometimes & 49 & 31.6 & $24.3-38.9$ \\
\hline No & 97 & 62.6 & $55.0-70.2$ \\
\hline \multicolumn{4}{|l|}{ Ever used drugs before } \\
\hline Yes & 5 & 3.2 & $0.4-6.0$ \\
\hline No & 141 & 91 & $86.5-95.5$ \\
\hline Not sure/unknown & 9 & 5.8 & $2.1-9.5$ \\
\hline \multicolumn{4}{|l|}{ Had sex before } \\
\hline Yes & 51 & 32.9 & $25.5-40.3$ \\
\hline No/no answer & 104 & 67.1 & $59.7-74.5$ \\
\hline \multicolumn{4}{|l|}{ Sexual debut $<$ age 15} \\
\hline Yes & 8 & 15.7 & $10.0-21.4$ \\
\hline No & 43 & 84.3 & $78.6-90.0$ \\
\hline \multicolumn{4}{|l|}{ Who were sex partner } \\
\hline Love partner & 45 & 88.2 & $83.1-93.3$ \\
\hline Other & 6 & 11.8 & $6.7-16.9$ \\
\hline \multicolumn{4}{|l|}{ Number of sex partners } \\
\hline One & 34 & 64.7 & $57.2-72.2$ \\
\hline Two or more & 17 & 35.3 & $27.8-42.8$ \\
\hline \multicolumn{4}{|l|}{ Condom use } \\
\hline Almost all the time & 24 & 47.1 & $39.2-55.0$ \\
\hline$>$ half of the time & 6 & 11.8 & $6.7-16.9$ \\
\hline$<$ half of the time & 8 & 15.7 & $10.0-21.4$ \\
\hline Never used condom & 13 & 25.4 & $18.5-32.3$ \\
\hline Age 21 - $24(n=93)$ & $\mathrm{N}$ & $\%$ & $95 \%$ CI \\
\hline \multicolumn{4}{|l|}{ Ever smoked before: } \\
\hline Yes & 51 & 54.8 & $44.7-64.9$ \\
\hline No/unknown & 42 & 45.2 & $35.1-55.3$ \\
\hline \multicolumn{4}{|l|}{ Smoked last year } \\
\hline Yes & 45 & 48.4 & $38.2-58.6$ \\
\hline No & 48 & 51.6 & $41.4-61.8$ \\
\hline \multicolumn{4}{|l|}{ Ever drank before } \\
\hline Yes & 69 & 74.2 & $65.3-83.1$ \\
\hline No & 24 & 25.8 & $16.9-34.7$ \\
\hline \multicolumn{4}{|l|}{ Drank last year } \\
\hline Yes & 29 & 31.2 & $21.8-40.6$ \\
\hline No & 64 & 68.8 & $59.4-78.2$ \\
\hline \multicolumn{4}{|l|}{ Ever got drunk } \\
\hline Yes all the time & 7 & 7.5 & $2.1-12.9$ \\
\hline Yes sometimes & 37 & 39.8 & $29.9-49.7$ \\
\hline No & 49 & 52.7 & $42.6-62.8$ \\
\hline \multicolumn{4}{|l|}{ Ever used drugs before } \\
\hline Yes & 6 & 6.5 & $1.5-11.5$ \\
\hline
\end{tabular}

\section{Continued}

\begin{tabular}{|c|c|c|c|}
\hline No & 84 & 90.3 & $84.3-96.3$ \\
\hline Not sure/unknown & 3 & 3.2 & $0.7-9.1$ \\
\hline \multicolumn{4}{|l|}{ Had sex before } \\
\hline Yes & 58 & 62.4 & $52.6-72.2$ \\
\hline No/no answer & 35 & 37.6 & $27.8-47.4$ \\
\hline \multicolumn{4}{|l|}{ Sexual debut < age 15} \\
\hline Yes & 9 & 15.5 & $8.1-22.9$ \\
\hline No & 49 & 84.5 & $77.1-91.9$ \\
\hline \multicolumn{4}{|l|}{ Who were sex partner } \\
\hline Love partner & 53 & 91.4 & $85.7-97.1$ \\
\hline Other & 5 & 8.6 & $2.9-14.3$ \\
\hline \multicolumn{4}{|l|}{ Number of sex partners } \\
\hline One & 42 & 72.4 & $63.3-81.5$ \\
\hline Two or more & 16 & 27.6 & $18.5-36.7$ \\
\hline \multicolumn{4}{|l|}{ Condom use } \\
\hline Almost all the time & 23 & 39.7 & $29.8-49.6$ \\
\hline$>$ half of the time & 14 & 24.1 & $15.4-32.8$ \\
\hline$<$ half of the time & 9 & 15.5 & $8.1-22.9$ \\
\hline Never used condom & 12 & 20.7 & $12.5-28.9$ \\
\hline Total & $\mathrm{N}$ & $\%$ & $95 \%$ CI \\
\hline \multicolumn{4}{|l|}{ Ever smoked before: } \\
\hline Yes & 126 & 50.8 & $44.4-57.2$ \\
\hline No/unknown & 122 & 49.2 & $42.8-55.6$ \\
\hline \multicolumn{4}{|l|}{ Smoked last year } \\
\hline Yes & 121 & 48.7 & $42.3-55.1$ \\
\hline No & 127 & 51.3 & $44.9-57.7$ \\
\hline \multicolumn{4}{|l|}{ Ever drank before } \\
\hline Yes & 162 & 65.3 & $59.2-71.4$ \\
\hline No & 86 & 34.7 & $28.6-40.8$ \\
\hline \multicolumn{4}{|l|}{ Drank last year } \\
\hline Yes & 72 & 29 & $23.2-34.8$ \\
\hline No & 176 & 71 & $65.2-76.8$ \\
\hline \multicolumn{4}{|l|}{ Ever got drunk } \\
\hline Yes all the time & 16 & 6.5 & $3.4-9.6$ \\
\hline Yes sometimes & 86 & 34.6 & $28.5-40.7$ \\
\hline No & 146 & 58.9 & $52.6-65.2$ \\
\hline \multicolumn{4}{|l|}{ Ever used drugs before } \\
\hline Yes & 11 & 4.5 & $1.9-7.1$ \\
\hline No & 225 & 90.7 & $87.0-94.4$ \\
\hline Not sure/unknown & 12 & 4.8 & $2.1-7.5$ \\
\hline \multicolumn{4}{|l|}{ Had sex before } \\
\hline Yes & 109 & 44 & $37.7-50.3$ \\
\hline No/no answer & 140 & 56 & $49.7-62.3$ \\
\hline \multicolumn{4}{|l|}{ Sexual debut < age 15} \\
\hline Yes & 17 & 15.6 & $11.0-20.2$ \\
\hline No & 92 & 84.4 & $79.8-89.0$ \\
\hline \multicolumn{4}{|l|}{ Who were sex partner } \\
\hline Love partner & 98 & 89.9 & $86.1-93.7$ \\
\hline Other & 11 & 10.1 & $6.3-13.9$ \\
\hline \multicolumn{4}{|l|}{ Number of sex partners } \\
\hline One & 76 & 69.7 & $63.8-75.6$ \\
\hline Two or more & 33 & 30.3 & $24.4-36.2$ \\
\hline \multicolumn{4}{|l|}{ Condom use } \\
\hline Almost all the time & 47 & 43.1 & $36.8-49.4$ \\
\hline$>$ half of the time & 20 & 18.3 & $13.4-23.2$ \\
\hline$<$ half of the time & 17 & 15.6 & $11.0-20.2$ \\
\hline Never used condom & 25 & 23.0 & $17.6-28.4$ \\
\hline
\end{tabular}


Table 4. Knowledge about HIV transmission and access to services and educational programs.

\begin{tabular}{|c|c|c|c|}
\hline Age $12-20(n=155)$ & $\mathrm{N}$ & $\%$ & $95 \% \mathrm{CI}$ \\
\hline Know about VCT: Yes & 22 & 14.2 & $8.7-19.7$ \\
\hline No & 133 & 85.8 & $80.3-91.3$ \\
\hline Have had VCT: Yes & 19 & 12.3 & $7.1-17.5$ \\
\hline No & 136 & 87.7 & $82.5-92.9$ \\
\hline HIV can be transmitted: Yes & 153 & 98.7 & $95.5-99.8$ \\
\hline No & 2 & 1.3 & $0.2-4.6$ \\
\hline Can HIV cause death: Yes & 133 & 85.8 & $80.3-91.3$ \\
\hline No & 22 & 14.2 & $8.7-19.7$ \\
\hline \multicolumn{4}{|l|}{ HIV transmission paths } \\
\hline Know at least one path & 138 & 89 & $84.1-93.9$ \\
\hline Know none & 17 & 19 & $12.8-25.2$ \\
\hline Know all four paths: Yes & 29 & 18.7 & $12.6-24.8$ \\
\hline No & 126 & 81.3 & $75.2-87.4$ \\
\hline Received HIV education: Yes & 115 & 74.2 & $67.3-81.1$ \\
\hline No & 40 & 25.8 & $18.9-32.7$ \\
\hline \multicolumn{4}{|l|}{ Source of information } \\
\hline TV & 119 & 76.8 & $70.2-83.4$ \\
\hline Friends & 45 & 29 & $21.9-36.1$ \\
\hline Internet & 60 & 38.7 & $31.0-46.4$ \\
\hline Radio & 71 & 45.8 & $38.0-53.6$ \\
\hline Posters & 55 & 35.5 & $28.0-43.0$ \\
\hline Pictures & 43 & 27.7 & $20.7-34.7$ \\
\hline HV education necessary: Yes & 132 & 85.2 & $79.6-90.8$ \\
\hline No & 23 & 14.8 & $9.2-20.4$ \\
\hline Age $21-24(n=93)$ & $\mathrm{N}$ & $\%$ & $95 \%$ CI \\
\hline Know about VCT: Yes & 27 & 29 & $19.8-38.2$ \\
\hline No & 66 & 71 & $61.8-80.2$ \\
\hline Have had VCT: Yes & 18 & 19.4 & $11.4-27.4$ \\
\hline No & 75 & 80.6 & $72.6-88.6$ \\
\hline HIV can be transmitted: Yes & 92 & 98.9 & $94.2-99.9$ \\
\hline No & 1 & 1.1 & $0.0-5.8$ \\
\hline Can HIV cause death: Yes & 81 & 87.1 & $80.3-93.9$ \\
\hline No & 12 & 12.9 & $6.1-19.7$ \\
\hline \multicolumn{4}{|l|}{ HIV transmission paths } \\
\hline Know at least one path & 87 & 93.5 & $88.5-98.5$ \\
\hline Know none & 6 & 6.5 & $1.5-11.5$ \\
\hline Know all four paths: Yes & 20 & 21.5 & $13.2-29.8$ \\
\hline No & 73 & 78.5 & $70.2-86.8$ \\
\hline Received HIV education: Yes & 63 & 67.7 & $58.2-77.2$ \\
\hline No & 30 & 32.3 & $22.8-41.8$ \\
\hline \multicolumn{4}{|l|}{ Source of information } \\
\hline TV & 69 & 74.2 & $65.3-83.1$ \\
\hline Friends & 41 & 44.1 & $34.0-54.2$ \\
\hline Internet & 28 & 30.1 & $20.8-39.4$ \\
\hline Radio & 30 & 32.3 & $22.8-41.8$ \\
\hline Posters & 40 & 43 & $32.9-53.1$ \\
\hline Pictures & 35 & 37.6 & $27.8-47.4$ \\
\hline HV education necessary: Yes & 81 & 87.1 & $80.3-93.9$ \\
\hline No & 12 & 12.9 & $6.1-19.7$ \\
\hline Total & $\mathrm{N}$ & $\%$ & $95 \% \mathrm{CI}$ \\
\hline Know about VCT: Yes & 49 & 19.8 & $14.8-24.8$ \\
\hline No & 199 & 80.2 & $75.2-85.2$ \\
\hline Have had VCT: Yes & 37 & 14.9 & $10.5-19.3$ \\
\hline
\end{tabular}

\section{Continued}

\begin{tabular}{lccc}
\hline No & 211 & 85.1 & $80.7-89.5$ \\
HIV can be transmitted: Yes & 245 & 98.8 & $96.5-99.7$ \\
No & 3 & 1.2 & $0.3-3.5$ \\
Can HIV cause death: Yes & 214 & 86.3 & $82.0-90.6$ \\
$\quad$ No & 34 & 13.7 & $9.4-18.0$ \\
HIV transmission paths & & & \\
Know at least one path & 225 & 90.7 & $87.1-94.3$ \\
Know none & 23 & 9.3 & $5.7-12.9$ \\
Know all four paths: Yes & 49 & 19.8 & $14.8-24.8$ \\
No & 199 & 80.2 & $75.2-85.2$ \\
Received HIV education: Yes & 178 & 71.8 & $66.2-77.4$ \\
No & 70 & 28.2 & $22.6-33.8$ \\
Source of information & & & \\
TV & 188 & 75.8 & $70.5-81.1$ \\
Friends & 86 & 34.7 & $28.8-40.6$ \\
Internet & 88 & 35.5 & $29.5-41.5$ \\
Radio & 101 & 41.7 & $35.6-47.8$ \\
Posters & 95 & 38.3 & $32.2-44.4$ \\
Pictures & 78 & 31.5 & $25.7-37.3$ \\
HV education necessary: Yes & 213 & 85.9 & $81.6-90.2$ \\
No & 35 & 14.1 & $9.8-18.4$ \\
\hline
\end{tabular}

15; the corresponding percentages for the age $20-24$ group are $62.4 \%$ and $15.5 \%$. In the $12-20$ age group, $35.3 \%$ had multiple sexual partners, compared to $27.6 \%$ of the 21 - 24 year olds. Despite of the fairly high prevalence of sexual activity, $23 \%$ of the all respondents had never used condoms, more so for the 12 - 20 year olds (25.4\%) than the older age group (20.7\%).

\subsection{Knowledge of HIV Transmission and Access to Services}

The vast majority of the respondents from both age groups understood that HIV can be transmitted $(98.7 \%$ $98.9 \%)$ and that it can lead to death (85.8\% - 87.1\%), and the majority were also knowledgeable about one of the four paths of transmission (89.0\% - 93.5\%) (Table 4). However, less than $20 \%$ of the younger age group (18.7\%) and about $22 \%$ of the older age group knew about all paths of HIV transmission.

The majority of the respondents (67.7\% to $74.2 \%)$ reported that they had received education about HIV but the sources of information about HIV varied considerably. About $70 \%$ of the respondents reported TV as a source of information on HIV transmission and prevention (see last panel of Table 4), and a significant proportion of the respondents obtained the information from friends (34.7\%), internet (35.5\%), radio (41.7\%), posters (38.3\%) and pictures (31.5\%). It is interesting to note that in the younger age group TV, internet and radio were more commonly used as a source of information than in the older age group who were more likely to report friends, posters and pictures as a source of information 
about HIV. The older age group was more likely to report that they knew about the voluntary counselling testing for HIV (29\%) and had used such service (19.4\%) than the younger age group (14.2\%, 12.3\% respectively).

\section{Discussion}

The data show that socially disadvantaged adolescents and youth were overrepresented in the out-of-school young people in Yunnan. More than 55\% of them were of national minorities (which make up only about 30\% of Yunnan's total population) and 70\% residing in rural areas, compared to $36 \%$ of the out-of-school youth in Anshan City in Liaoning Province [14]. More than 45\% of the out-of-school young people came from families where the parents had no schooling or only primary schooling. More than $50 \%$ of the youth left school for reasons other than school completion and they relied on themselves, friends or others for financial support.

The out-of-school young people in Yunnan live in the epicentre of drug abuse of China, with a high level of exposure to illicit drugs. By the end of 2008 there were 99,000 registered drug users in Yunnan, making $0.2 \%$ of the total population (42.36 million) [3]. However, more than 20 times as many respondents in our study population $(4.4 \%)$ reported that their parents used illicit drugs, and $4.5 \%$ of them used drugs themselves also, a prevalence that is also higher than that in out-of-school youth in Anshan, Liaoning Province (3.8\%) [14]. About $20 \%$ of the respondents reported that their friends used drugs. The true prevalence of drug use in the study population could be higher because $4.8 \%$ of the respondents were not sure if they had ever used drugs, which could reflect a reluctance to admit using drugs due to social undesirability or stigma [15].

Our findings show that $\mathrm{K}$ power, ecstasy, ice and opium have become much more commonly used than heroin now in Yunnan. These drugs are relatively new to the Chinese setting. Curiosity and lack of knowledge about the harm of abusing new drugs are likely to be the main reasons for increasing number of youth initiating these drugs. Use of some of these new drugs not only causes physical and psychological harms but it also reduces sexual inhibition and increases unprotected sexual activities [16], a primary risk factor for HIV transmission. Being drug users and being sexual active without protection places some out-of-school youth at double risks for HIV transmission. Hence prevention of drug abuse should be a critical element of HIV education and prevention.

Sexual contact has become a major path of HIV transmission in Yunnan. Based on official figures from January to September 2009, 64.5\% of HIV infections were transmitted through sex [17]. Our findings have shown that more than $44 \%$ of the out-of-school adolescents and youth in Yunnan had sex prior to the survey, and a considerable proportion of them had sexual debut at or below age 15. Having multiple sexual partners and never using condom was more common in the younger age group (35.3\%, 25.4\% respectively) than in the older age group (27.6\% and $20.7 \%$, respectively). The high prevalence of unsafe sex in the out-of-school young people in Yunnan may be attributed to several factors. Many of this population migrated from rural areas to cities to look for work. Being a transition population, lack of social and economic support, isolation and lack of adequate knowledge about HIV or of access to condoms may have lead them into unsafe sex as a way of finding stimulation and emotional support.

Despite that the vast majority of the study population reported to have received education about HIV transmission and prevention (72\%), only about 20\% knew all transmission paths or knew about the VCT, and less than $15 \%$ of them had ever used the VCT service. This suggests that future HIV education programs need to target this vulnerable population with much deeper knowledge about HIV transmission and prevention and harms associated with drug use, particularly drugs that are new to the Chinese setting. Our results show that there are multiple channels through which such knowledge can be disseminated, including television, internet, radio, posters/pictures and friends. The use of television, internet and radio has become a more common source of information for the younger than the older age group. It is likely that these media will continue to gain popularity in the future as a way of reaching the out-of-school youth for HIV education.

In summary, the out-of-school young people in Yunnan exhibited similar vulnerability to HIV as found in out-of-school young people in Beijing [9,11], Liaoning Province (North China) [14], and Hunan Province (Central China) [18]: lack or limited knowledge about HIV transmission and prevention, a high prevalence of sexual activity with multiple partners but with low levels of condom use. In addition, the out-of-school young people in Yunnan demonstrated a high exposure to illicit drugs and a high prevalence of drug use amongst themselves, as also found in other high HIV risk areas of China (e.g., Guizhou and Guangxi) [19,20]. Further, our study has shown an important trend: the exposure to parental drug use and alcohol abuse, to heroin and ecstasy, the prevalence of having multiple sexual partners and non-use of common is higher in younger than older out-of-school young people.

\section{Conclusion}

With increasing difficulties in finding employment in 
university graduates in recent years in China [21] and increases in school-age migrants into urban areas [22], the number of out-of-school adolescents and youth who drift into the margin of society will increase. Reaching them for HIV education represents a great challenge as this is a highly mobile population in terms of residence and work, with tenuous connections to, the family, community and the labor market. As our study has shown, use of multiple media, particularly television, internet and radio, is likely to be an effective way of providing this population with adequate education about HIV prevention.

\section{REFERENCES}

[1] China News Network Beijing, "Overview of the Current Situation of the Global HIV/AIDS Epidemic," 2008. http://news.xinhuanet.com/world/2008-08/06/content_89 98322.htm

[2] Joint United Nations Programme on HIV/AIDS, China Ministry of Health and World Health Organization, "Joint Review Report of HIV/AIDS Prevention in China,” 2007. http://www.gov.cn/xwfb/2007-11/29/content_820426.htm

[3] China Ministry of Health, Joint United Nations Programme on HIV/AIDS, and World Health Organization, "An Assessment of China's HIV/AIDS Epidemic in 2011,” 2011.

http://www.moh.gov.cn/publicfiles/business/htmlfiles/mo hjbyfkzj/s3586/201201/53957.htm

[4] Y. Chen and Q. Li, "Yunnan Provincial Report: Cumulative HIV/AIDS Cases Reach 63,322,” 2008.

http://www.yn.xinhuanet.com/newscenter/2008-11/27/co ntent_15032917.htm

[5] Yunnan Provincial Centre for Disease Control and Prevention, 2009 (Unpublished material).

[6] L. Yang, J. Li, Y. Zhang, W. Zhang, F. Dai and B. Maycock, "Reported Reasons for Initiating Drug Use among DrugDependent Adolescents and Youth in Yunnan, China," The American Journal of Drug and Alcohol Abuse, Vol. 35, No. 6, 2009, pp. 445-453. doi:10.3109/00952990903469299

[7] Y. Fu and Q. Gao, "Summary Report of the Development of Science, Technology and Education in West China," 2005.

http://www.china.com.cn/zhuanti2005/txt/2005-11/03/con tent_6019101.htm

[8] Y. Ma, "Strategies for Reducing Vulnerability to HIV/ AIDS in Adolescents and Youth," Chinese Journal of Reproductive Health, Vol. 19, No. 3, 2008, pp. 139-143.

[9] C. Wang, Y. Ma and S. Ding, "Analysis of HIV Vulnerability in Out-of-School Adolescents and Youth in Beijing," Chinese School Health, Vol. 8, No. 10, 2007, pp. 873-877.

[10] C. Chang, L. Chen, X. Sun, L. Zhang, D. Tian and X. Zhang, "A Holistic Approach to Analysing HIV Vulnera- bility in Out-of-School Young People,” Beijing University Journal (Medicine Section), Vol. 39, No. 3, 2007, pp. 132-135.

[11] Y. Ma, S. Ding and C. Wang, "HIV/AIDS Vulnerability and Contributing Factors in Out-of-School Migrant School Age Children in Beijing," Beijing University Journal (Medicine Section), Vol. 39, No. 2, 2007, pp. 32-135.

[12] B. Dick, J. Ferguson and D. A. Ross, "Preventing HIV/ AIDS in Young People: A Systematic Review of the Evidence from Developing Countries," WHO Technical Report Series 938, 2006, pp. 1-2.

[13] Encyclopædia Britannica, Inc., "Britannica Concise Encyclopedia,” 1994-2008. http://encyclopedia2.thefreedictionary.com/adolescence

[14] H. Hu, L. Chen and C. Chang, "High HIV Risky Behaviours in Out-of-School Adolescents and Youth and Contributing Social Factors,” Journal of Disease Control, Vol. 4, No. 11, 2007, pp. 333-335.

[15] R. Deng, J. Li, L. Sringernyuang and K. Zhang, "Drug Abuse, HIV/AIDS, and Stigmatisation in a Chinese Indigenous Community," Social Science \& Medicine, Vol. 64, No. 8, 2007, pp. 1560-1571. doi:10.1016/j.socscimed.2006.12.011

[16] N. Wang, "Drug Use Is the Main Contributing Factor for HIV Transmission in China and Abuse of New Illicit Drugs Leads to Sexual Promiscuity,” 2008.

http://www.china.com.cn/news/2008-12/01/content_1687 9079.htm

[17] F. Liu and C. Zhou, "Yunnan Dali Gay Men Night Club Incident: Most Homosexual Men Are Located in Rural Areas,” 2009. http://health.sohu.com/20091221/n269088061.shtml

[18] S. Teng, H. Peng, M. Luo and X. Tang, “A Study of Knowledge about HIV/AIDS in Rural Out-of-School Adolescents and Youths," Practical Preventive Medicine, Vol. 12, No. 6, 2005, pp. 1389-1390.

[19] C. Long, “An Investigation of High Risk Behaviour and HIV Vulnerability in Adolescents and Youth outside School in Guizhou Province,” Northwest Population, Vol. 28, No. 6, 2007, pp. 95-101.

[20] S. Luo, "On the High Risk Behaviours of Out-of-School Youngsters and Their Vulnerability to HIV/AIDS,” Journal of Guangxi University for Minority Nationalities (Medical Anthropology Edition), Vol. 28, No. 3, 2006, pp. 39-42.

[21] C. Dong, "Substantial Decrease in the Employment Rate amongst More than 100,000 University Graduates Sincerely Yours, the End of Last Year,” 2009. http://news.163.com/09/0628/11/5CT1QAFU000120GR. html

[22] Z. Zhang and J. He, "Difficulties for University Graduates to Find Work and for Employers to Recruit Workers in Wuhan Province,” 2008. http://news.sohu.com/20081112/n260599526.shtml 